\title{
Neutrophil sensing of cytoplasmic, pathogenic DNA in a cGAS-STING-independent manner
}

\author{
Zhou Yu ${ }^{1}$, Taoyong Chen ${ }^{2}$ and Xuetao Cao ${ }^{1,2,3}$ \\ Cellular \& Molecular Immunology (2016) 13, 411-414;doi:10.1038/cmi.2015.34;published online 27 April 2015
}

The innate immune system is an integral part of the host response to invading pathogens and endogenous danger signals. Pattern recognition receptors (PRRs), which are utilized by the innate immune system, can detect pathogen-associated molecular patterns (PAMPs) of invading pathogens and damage-associated molecular patterns (DAMPs) and signal to initiate the innate immune response. ${ }^{1,2}$ Upon recognition by PRRs, the receptor activates downstream signaling pathways, leading to the production of proinflammatory cytokines and type I interferons (IFNs), which function to eliminate the invading pathogens or, alternatively, cause inflammatory and autoimmune diseases. ${ }^{1,2}$ Nucleic acids, which exist in all living organisms, are important danger signals when detected by the innate immune system in certain unfamiliar locations or chemical states. PRRs have been implicated in the detection of RNA and DNA derived from both pathogens and hosts; examples include Toll-like receptors (TLRs), RIG-I-like receptors (RLRs), AIM2-like receptors (ALRs), and NOD-like receptors (NLRs). ${ }^{1,2}$ Understanding the mechanisms that govern immune cell recognition of pathogenic DNA is crucial for elucidating the anti-microbial and autoimmune responses.

A series of cytosolic DNA sensors have been uncovered, such as cyclic GMP-AMP (cGAMP) synthase (cGAS), DNAdependent activator of IFN regulatory factors (DAI; also known as ZBP1 or DLM1), IFN-inducible protein (IFI16), DExD/H family of helicase (DDX41), absent in melanoma 2 (AIM2), leucine-rich repeat (in Flightless I) interacting protein-1 (LRRIFIP1), DNA-dependent protein kinase (DNA-PK), and meiotic recombination 11 homolog A (MRE11). ${ }^{1-3}$ Most of these identified DNA sensors can bind DNA and initiate the production of type I IFNs. ${ }^{1,2}$ However, AIM2 has been implicated in the regulation of inflammasome activation and IL- $1 \beta$ maturation. ${ }^{4-6}$ IFI16 is a special DNA sensor that can activate both inflammasome-mediated IL- $1 \beta$ maturation and STINGdependent type I IFNs production (STING: stimulator of IFNs; also known as TMEM173, MITA, ERIS and MPYS) ${ }^{7,8}$ Despite the many DNA sensors that have been identified, cGAS-STING is thought to be the core machinery governing DNA sensing and signaling. In cells, cGAS-STING mediates the activation of TANK-binding kinase 1 (TBK1) and IFN regulatory factor IRF3 (Figure 1). ${ }^{9-11}$ Pathogenic DNA is detected by cGAS, which induces the synthesis of cGAMP; subsequently, cGAMP acts as a second messenger that associates with and activates STING. ${ }^{9} 10$ STING has been implicated as a central player in DNA-induced type I IFN production. ${ }^{11}$ The mechanism by which STING mediates the production of inflammatory cytokines is not completely understood. It is also uncertain whether there are cGAS-STING-independent pathways in the host-cell innate immune response for DNA sensing and signaling for type I IFN production.

The major cellular models used in studies of DNA sensors include macrophages, mouse embryonic fibroblasts, dendritic cells (DCs), and fibroblasts. ${ }^{1,2}$ Neutrophils are the most abundant type of white blood cells in mammals and are usually the first responders at sites of infection or injury. ${ }^{12}$ The recognition of PAMPs and subsequent phagocytosis of microorganisms by neutrophils can involve the expression of multiple TLRs (with the exception of TLR3), RLRs (RIG-I and MDA5), NOD1, C-type lectin receptors, and complement receptors. ${ }^{12}$ It has long been recognized that neutrophils play a pivotal role in anti-bacterial and anti-fungal innate immunity, possibly via the production of reactive oxygen species and the release of antimicrobial peptides and protein-decorated chromatin, also known as neutrophil extracellular traps. ${ }^{12}$ However, neutrophilic sensing of viral nucleic acids is not clearly understood; particularly, it remains unknown whether neutrophils are equipped with DNA sensors and whether cGAS-STING is used in signal mediation.

A study by Dr Z. Fan and colleagues which was published in a recent issue of Nature Immunology identified SOX2 as a sensor

${ }^{1}$ Institute of Immunology, Zhejiang University School of Medicine, Hangzhou, China; ${ }^{2}$ National Key Laboratory of Medical Immunology \& Institute of Immunology, Second Military Medical University, Shanghai, China and ${ }^{3}$ National Key Laboratory of Medical Molecular Biology \& Department of Immunology, Institute of Basic Medical Sciences, Chinese Academy of Medical Sciences, Beijing, China

Correspondence: Dr Xuetao Cao, Institute of Immunology, Zhejiang University School of Medicine, Hangzhou 310058, China. E-mail: caoxt@immunol.org Received: 18 March 2015; accepted: 18 March 2015 


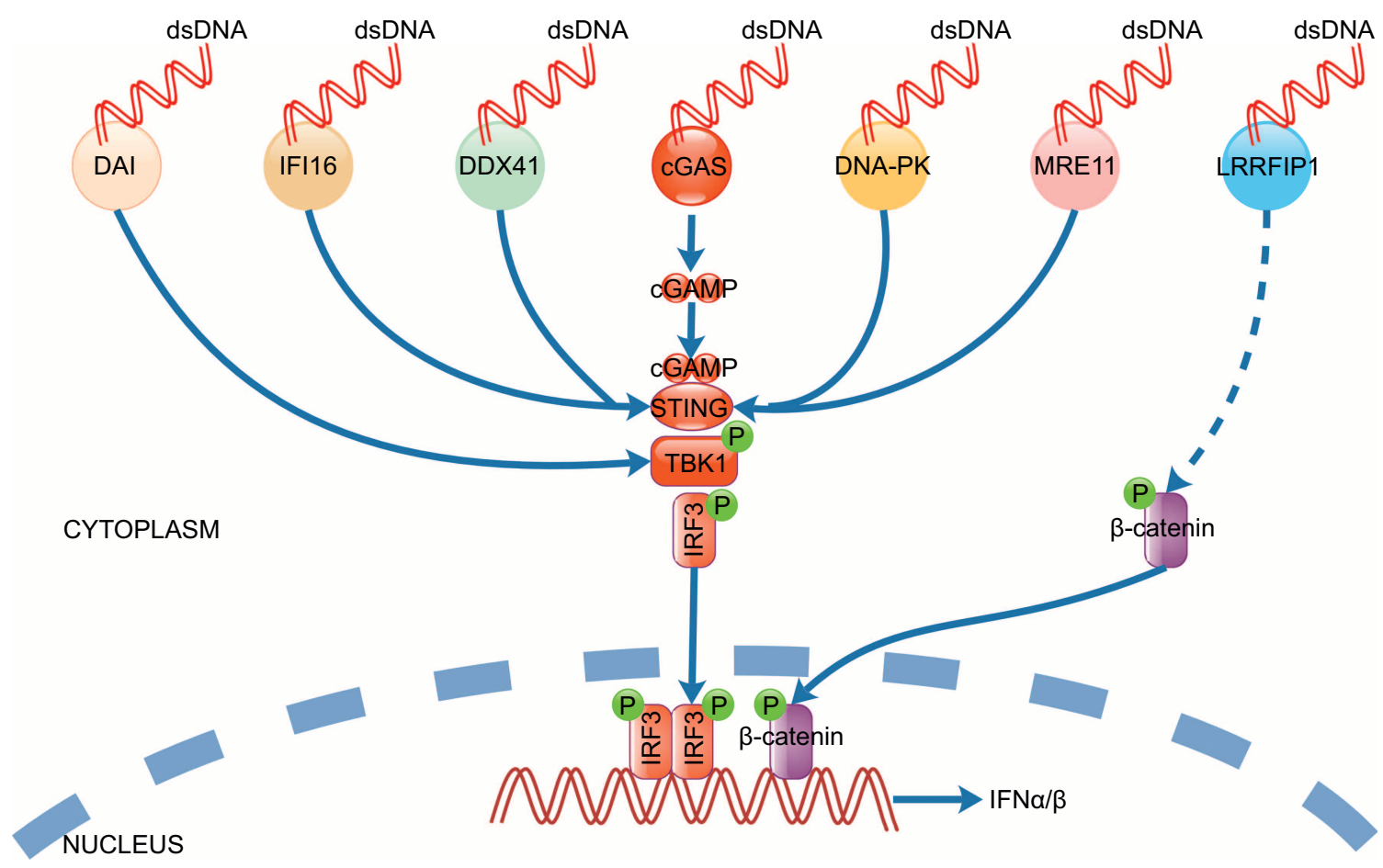

Figure 1 Cytoplasmic DNA sensors involved in DNA sensing and type I IFN induction. DNA sensors that are not illustrated in this diagram include the inflammasome-activating AIM2, RNA polymerase II, which transcribes dsDNA into sSRNA and initiates RIG-I signaling, and the high-mobility group box proteins (HMGBs), which detect various suspicious nucleic acids. Abbreviations: cGAMP, cyclic GMP-AMP; cGAS, cGAMP synthase; DAI, DNA-dependent activator of IFN-regulatory factors; DDX41, DExD/H-Box helicase 41; DNA-PK, DNA-dependent protein kinase; dsDNA, doublestranded DNA; IFI16, interferon gamma-inducible protein 16; IRF3, interferon regulatory factor 3; LRRFIP1, leucine-rich repeat (in Flightless I) interacting protein-1; MRE11, meiotic recombination 11 homolog A; P, phosphonate; STING, stimulator of IFN genes; TBK1, TANK-binding kinase 1.

of pathogenic DNA that is present in neutrophils and triggers the activation of NF- $\kappa \mathrm{B}$ and $\mathrm{AP}-1$ transcription factors and the production of TNF $\alpha$, IL- 6 and IL-1 $\beta$ proinflammatory cytokines, as exemplified in the inhibition of Listeria monocytogenes (LM) and Bartonella henselae infections (Figure 2). ${ }^{13}$ This study defined the important machinery involved in sensing pathogenic DNA in neutrophils that express extremely low levels of cGAS and STING, thereby casting new light on the mechanism of recognition of pathogenic DNA by neutrophils.

SOX2 is a transcription factor that is essential for maintaining the self-renewal and pluripotent capacities of undifferentiated embryonic stem cells. The study from the group of Dr Fan investigated the function of SOX2 in immune cells ${ }^{13}$ by first examining SOX2 expression in mature hematopoietic cells, discovering that SOX2 is expressed in only neutrophils. SOX2 localized to the cytoplasm in mouse and human neutrophils, which suggested a special role of SOX2 in neutrophils, particularly in the innate immune response. The authors infected the neutrophil-specific SOX2-deficient mice with LM and found that the survival rate and the concentrations of proinflammatory cytokines (TNF $\alpha$, IL- 6 , IL-1 $\beta$ ) in the serum of the SOX2-deficient mice were both significantly lower than those of the control group. To assess whether cGAS-STING is involved in this process, the authors utilized STING-deficient neutrophils and found that the production of proinflammatory cytokines was not impaired and the formation of the cGAS-STING-activated IRF3 dimer was not observed in wild-type neutrophils after transfection with LM DNA. Additionally, cGAS expression cannot be detected in neutrophils. Cumulatively, these findings indicate that SOX2 plays a critical role in neutrophil-mediated bacterial clearance and proinflammatory cytokine production in a manner that is likely independent of the cGAS-STING DNA sensing pathway.

SOX2 is a transcription factor containing a highly conserved DNA-binding domain known as the high-mobility group (HMG)-box domain. ${ }^{13}$ The authors hypothesized that cytosolic SOX2 in neutrophils might sense bacterial DNA. They searched for potential SOX2-binding regions in the LM genome by comparing the most well-defined SOX2-binding DNA sequence with the LM genomic DNA sequence, and the authors found that the matched sequences in LM genome were capable of binding the HMG-box domain of SOX2 in vitro. These results clearly identified the SOX2-binding motif in LM DNA; this discovery represents a great advancement in the field of DNA sensor proteins, in which very few of the recognized DNA sequences are known. ${ }^{1,2}$ The authors further found that TAB2 and TAK1 were the important interacting proteins functioning downstream of SOX2-DNA binding after bacterial infection. SOX2, which dimerized upon recognition of bacterial DNA, was essential for the dimerization of TAK1, the activation of the TAB2-TAK1 complex, and subsequent downstream signals. Thus, SOX2 may activate a TAB2-TAK1-dependent 


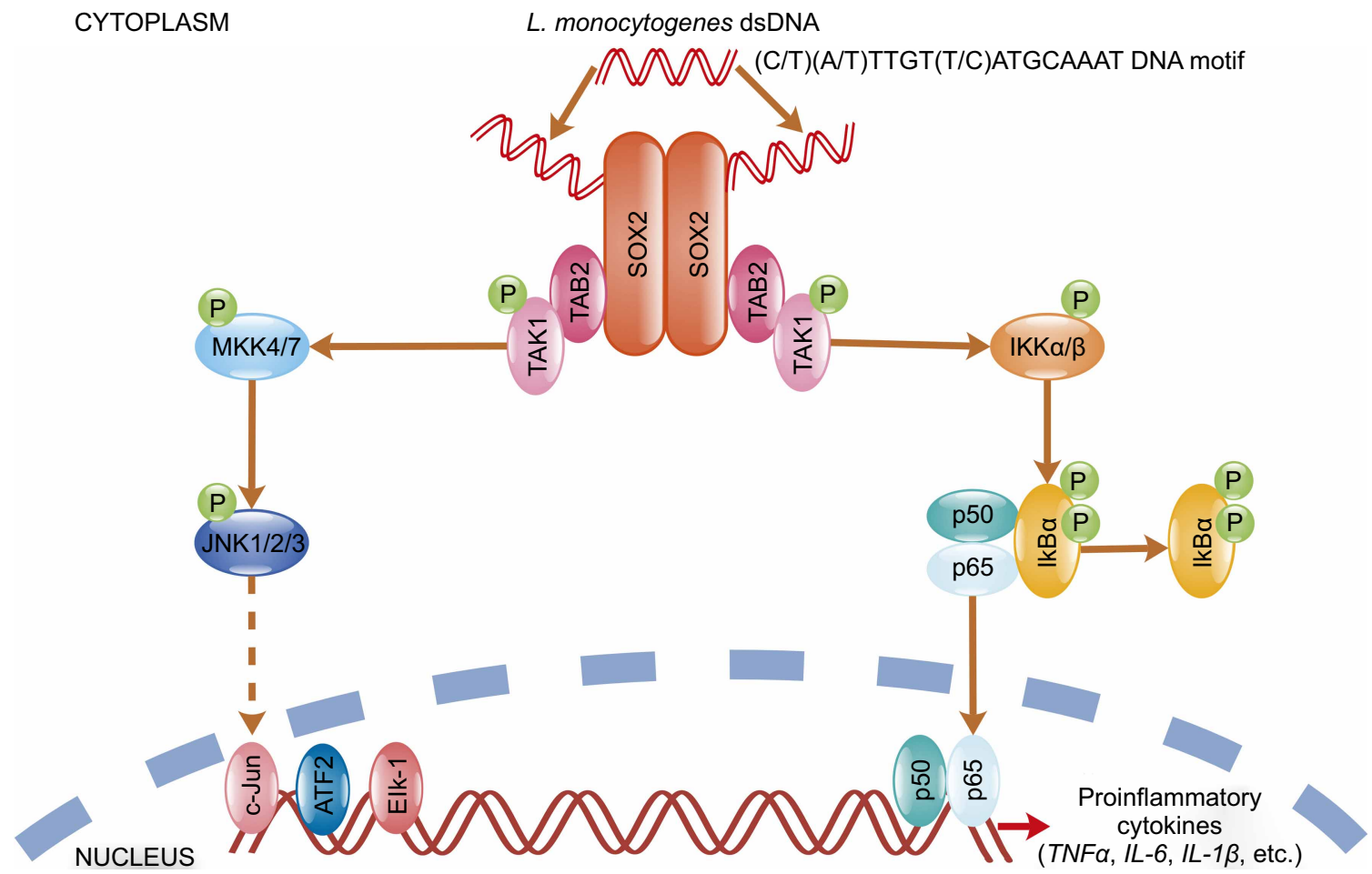

Figure 2 SOX2-mediated sensing of cytoplasmic DNA and the associated signaling pathway in neutrophils. After recognition of a specific DNA sequence in L. monocytogenes by the HMG domain in SOX2, SOX2 dimerizes and associates with TAB2 and TAK1, leading to dimerization and activation of TAK1. The TAK1 dimer then activates MKK4/7 and IKK $\alpha / \beta$, which phosphorylate JNK and IKB $\alpha$, respectively. JNK activates multiple transcription factors, such as AP-1, whereas $1 \kappa B \alpha$ phosphorylation releases the p50 and p65 transcription factors for import into the nucleus and

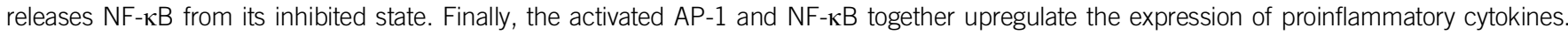

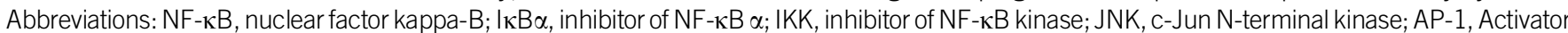
protein 1; MKK, mitogen-activated protein kinase; TAB2, TGF-beta activated kinase 1/MAP3K7 binding protein 2; TAK1, TGF-beta activated kinase 1.

signaling pathway for induction of proinflammatory cytokines in neutrophils upon recognition of bacterial DNA. Finally, the authors clarified that the TAB2-TAK1 complex plays a nonredundant role in the SOX2-mediated innate immune response using mice and neutrophils deficient in TAB2 or TAK1. These data indicate that the TAB2-TAK1 complex is essential for antibacterial immunity and that its activation is mediated by SOX2DNA binding in neutrophils.

Findings presented in this paper open new avenues for research of cytosolic DNA sensors in neutrophils. However, a series of concerns have also been raised by this study. The specific DNA sequence recognized by $\mathrm{SOX} 2$ in the LM genome reportedly also exists in host genomic DNA. ${ }^{13}$ Thus, it is possible that host DNA released during injury or virus/bacteria-induced tissue damage may lead to the activation of the SOX2-TAB2-TAK1 pathway in neutrophils and activate the innate immune response, in which case SOX2 would not only be a bacterial DNA sensor but also an endogenous DNA sensor. It is therefore highly interesting to research the potential involvement of SOX2 in autoimmune diseases. Additionally, the role of TAK1 in the neutrophil-mediated innate immune response is controversial when considered in relation to previous research. ${ }^{11}$ Ajibade et al. identified a previously unrecognized role of TAK1 as a negative regulator of $\mathrm{p} 38$ and IKK activation upon interaction with TLR4 in neutrophils. ${ }^{14}$
It may be interesting to examine whether SOX2 and TLR4 cause different modifications of TAK1. Type I IFNs exert a variety of biological effects on the immune system, especially the innate anti-microbial innate processes. ${ }^{15}$ Most DNA sensors can initiate the production of type I IFNs. ${ }^{1,2}$ However, type I IFN production by neutrophils is not a major object of interest in this study because of the low levels of IFN $\alpha / \beta$ produced by neutrophils. ${ }^{13}$ Yet, considering the enormous number of neutrophils, the type I IFNs that are secreted by them may still be remarkable. Therefore, future studies that examine whether additional DNA sensors exist in neutrophils and whether these sensors elicit a type I IFN response independent of cGAS-STING would be worthwhile.

The work by Dr Fan's group has advanced the research of pathogenic DNA sensing by neutrophils. However, much remains to be investigated to reach a complete understanding of the innate response of neutrophils to pathogenic DNA. First, a major question that remains is whether the sequence recognized by SOX2 from the LM genome is similarly recognized when present in diverse viral genomes. Second, it will be important to determine whether other DNA sensors (such as DAI, IFI16, and AIM2) also function in neutrophils and whether their activation results in the production of type I IFNs or inflammatory mediators by neutrophils. Third, whether additional regions of the LM genome cause an 
immunogenic effect in neutrophils is not known. Fourth, it is of interest to determine whether the SOX2-mediated recognition of DNA triggers cell death pathways, a phenomenon which may have contributed to the in vivo effects observed in SOX2-deficient mice. Finally, it remains to be determined whether cGAS and STING are inducible in neutrophils during infection. All of these questions require further studies and indepth analyses of DNA sensor profiles in neutrophils, and these studies should include the use of more natural pathogens.

1 Paludan SR, Bowie AG. Immune sensing of DNA. Immunity 2013; 38:870-880.

$2 \mathrm{Wu} \mathrm{J}$, Chen ZJ. Innate immune sensing and signaling of cytosolic nucleic acids. Annu Rev Immunol 2014; 32: 461-488.

3 Yang P, An H, Liu X, Wen M, Zheng Y, Rui Y et al. The cytosolic nucleic acid sensor LRRFIP1 mediates the production of type I interferon via a beta-catenin-dependent pathway. Nat Immunol2010; 11: 487-494.

4 Hornung V, Ablasser A, Charrel-Dennis M, Bauernfeind F, Horvath G, Caffrey DR et al. AIM2 recognizes cytosolic dsDNA and forms a caspase1-activating inflammasome with ASC. Nature 2009; 458: 514-518.

5 Fernandes-Alnemri T, Yu JW, Datta P, Wu J, Alnemri ES. AIM2 activates the inflammasome and cell death in response to cytoplasmic DNA. Nature 2009; 458: 509-513.

6 Bürckstümmer T, Baumann C, BlümI S, Dixit E, Dürnberger G, Jahn H et al. An orthogonal proteomic-genomic screen identifies AIM2 as a cytoplasmic DNA sensor for the inflammasome. Nat Immunol 2009; 10: $266-272$.

7 Unterholzner L, Keating SE, Baran M, Horan KA, Jensen SB, Sharma $S$ et al. IFI16 is an innate immune sensor for intracellular DNA. Nat Immunol 2010; 11: 997-1004.

8 Kerur N, Veettil MV, Sharma-Walia N, Bottero V, Sadagopan S, Otageri $\mathrm{P}$ et al. IFI16 acts as a nuclear pathogen sensor to induce the inflammasome in response to Kaposi Sarcoma-associated herpesvirus infection. Cell Host Microbe 2011; 9: 363-375.

9 Sun L, Wu J, Du F, Chen X, Chen ZJ. Cyclic GMP-AMP synthase is a cytosolic DNA sensor that activates the type I interferon pathway. Science 2013; 339: 786-791.

10 Li XD, Wu J, Gao D, Wang H, Sun L, Chen ZJ. Pivotal roles of cGAScGAMP signaling in antiviral defense and immune adjuvant effects. Science 2013; 341: 1390-1394.

11 Ishikawa H, Ma Z, Barber GN. STING regulates intracellular DNAmediated, type I interferon-dependent innate immunity. Nature 2009; 461: 788-792.

12 Mantovani A, Cassatella MA, Costantini C, Jaillon S. Neutrophils in the activation and regulation of innate and adaptive immunity. Nat Rev Immunol 2011; 11: 519-531.

13 Xia P, Wang S, Ye B, Du Y, Huang G, Zhu P et al. Sox2 functions as a sequence-specific DNA sensor in neutrophils to initiate innate immunity against microbial infection. Nat Immunol 2015; 16 : 366-375.

14 Ajibade AA, Wang Q, Cui J, Zou J, Xia X, Wang M et al. TAK1 negatively regulates NF-kB and p38 MAP kinase activation in Gr-1+CD11b+ neutrophils. Immunity 2012; 36: 43-54.

15 Theofilopoulos AN, Baccala R, Beutler B, Kono DH. Type I interferons (alpha/beta) in immunity and autoimmunity. Annu Rev Immunol 2005; 23: 307-336. 